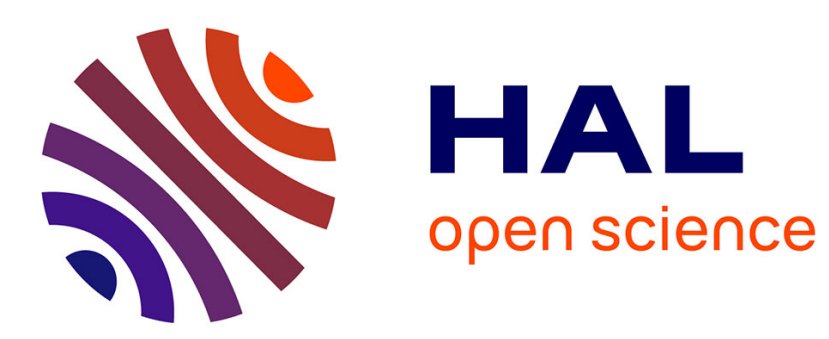

\title{
Réflexion sur le processus coracoïde des hominoïdes et des atèles ; application à quelques hominidés fossiles
}

Jean-Luc Voisin

\section{To cite this version:}

Jean-Luc Voisin. Réflexion sur le processus coracoïde des hominoïdes et des atèles; application à quelques hominidés fossiles. Comptes Rendus de l'Académie des Sciences - Series IIA - Earth and Planetary Science, 2001, 332, pp.229-306. halshs-00352057

\section{HAL Id: halshs-00352057 https://shs.hal.science/halshs-00352057}

Submitted on 12 Jan 2009

HAL is a multi-disciplinary open access archive for the deposit and dissemination of scientific research documents, whether they are published or not. The documents may come from teaching and research institutions in France or abroad, or from public or private research centers.
L'archive ouverte pluridisciplinaire HAL, est destinée au dépôt et à la diffusion de documents scientifiques de niveau recherche, publiés ou non, émanant des établissements d'enseignement et de recherche français ou étrangers, des laboratoires publics ou privés. 


\title{
Réflexion sur le processus coracoïde des hominoïdes et des atèles ; application à quelques hominidés fossiles
}

\author{
Jean-Luc Voisin* \\ Institut de Paléontologie Humaine, 1, rue René-Panhard, 75013 Paris, France
}

Reçu le 11 juillet 2000 ; accepté le 8 janvier 2001

Présenté par Yves Coppens

\begin{abstract}
Remark on the coracoid process of hominoids and spider monkeys, application to some hominid fossils. The orientation of the coracoid process, in relation to the scapula, is different in man on one hand and other hominoids (Pan, Gorilla, Pongo, Hylobates), spider and colobus monkeys on the other hand. On the contrary, the orientation of the coracoid process is similar in all hominoids and spider monkeys when it is taken in relation to the axial skeleton. Morphology of the coracoid process reveals the pattern of arm movements and some differences between modern human, Neandertal man and Homo ergaster. () 2001 Académie des sciences / Éditions scientifiques et médicales Elsevier SAS
\end{abstract}

coracoid process / hominoids / Ateles / Neandertal / Homo ergaster

Résumé - L'étude du processus coracoïde chez Homo, Pan, Gorilla, Pongo, Hylobates, Ateles et Colobus montre que son orientation est variable par rapport au corps de la scapula, mais qu'elle est constante par rapport au squelette axial. La morphologie du processus coracoïde reflète l'importance des mouvements du membre supérieur en dehors du plan parasagittal, ainsi que les différences entre les hommes modernes et des représentants plus anciens du genre Homo. ๑ 2001 Académie des sciences / Éditions scientifiques et médicales Elsevier SAS

processus coracoïde / hominoïdes / atèles / Néandertal / Homo ergaster

\section{Abridged version}

A lot of studies have been done about primates' scapulae $[1,3,6,13,16,18,21,25,27]$. However, apart from Martin and O'Brien's work [10], there are no studies about the coracoid process yet.

This work is a study of the orientation and morphology of the coracoid process in hominoids, spider monkeys, colobus monkeys, two neandertal and Homo ergaster remains (table I). Among fossil hominids, there are only four remains that still possess a coracoid process. Five points are used (figure 1). Three of them $(E, F, Q)$ define two perpendicular planes $P$ and $P^{\prime}$, and the two others $(U, R)$ define the great axis of the process. The projection of these five points in $P$ and $P^{\prime}$ planes allows to know the coracoid process orientation in comparison with the glenoid cavity (gleno-coracoid- $p$ and gleno-coracoid- $p^{\prime}$ angles). The coracoid process morphology is described by three indexes. The coracoid indices illustrate the length of the coracoid process and the two others (maximum and minimum robustness index) its breadth.

The gleno-coracoid- $p$ and the gleno-coracoid- $p^{\prime}$ angles show two groups. The first one, with the greatest value, contains only man. The second one includes all the other primates' studies here (figures 2 and 3, table II). The coracoid index has the greatest value in gibbons and spider monkeys (figure 4, table III), the most brachiating primates. The two coracoid robustness indices define also two groups, the spider monkey with the breadless coracoid process and the hominoids and the colobus monkey with a more robust coracoid process (figures 5 and 6, table III).

When the coracoid process orientation put in relation to the glenoid cavity, there is a great difference between man and the other hominoids and spider monkeys. On

* Correspondance et tirés à part.

Adresse e-mail : jeanlucv@mnhn.fr (J.-L. Voisin). 
the contrary, the coracoid process orientation seems to be similar between all the hominoids and spider monkey when it is taken in comparison with the axial skeleton (figure 7). In hominoids and spider monkeys, the scapula is dorsal, in comparison to the thorax, with its medial border parallel to the vertebral column when the shoulder is in rest position, that means with hanging arms in man [14-16]. In this position, the coracoid process is perpendicular to the vertebral column.

So, the differences observed on the gleno-coracoid- $p$ angle and gleno-coracoid- $p^{\prime}$ angle between man, other hominoids and spider monkeys reflect the difference of the glenoid cavity orientation between these two groups $[15,27]$. Values for the colobus monkeys show that their scapula may take a position similar to those of hominoids when they hang with their arms.

The coracoid process is longer in species that have a great use of arm movement outside the parasagittal plane. The exceptionally short orang-utan's coracoid process is difficult to interpret. It may be in relation to the peculiar locomotion and shoulder musculature of this species [23, 24]. The coracoid process elongation is not associated with the formation of the acromio-coracoid vault because it does not exist in Ateles and exists in Pongo [4]. The coracoid process length increases reinforce the leverage motion and removes muscle insertions away from the clavicle border. The straight morphology of the orang-utan clavicle [16, 28] could also explain the short length of their coracoid process. This is confirmed by the clavicle morphology of colobus monkeys [27].

The gleno-coracoid- $p$ and gleno-coracoid- $p^{\prime}$ angles of Kebara and Tabun CI are included in the interval of modern man (table $I V$ ). The peculiar values of these two angles for KNM-WT 15000 (table IV) could be explained by the loss of the growth cartilage [30]. The length of the coracoid process in Kebara, Tabun CI and KNM-WT 15000 are very close to those in modern man (table IV). These differences are of diagenetic nature. The coracoid processes of these three fossils shows a minimum robustness index inferior to those of modern man (table IV). On the contrary, the maximum robustness index of these three fossil men is higher than those of modern man (table IV). The peculiar coracoid process morphology of these three fossils is, possibly, a new criterion for distinguishing human fossils and modern human scapulae. However, more scapulae must be found before concluding definitively.

\section{Introduction}

De nombreuses études existent sur les scapulas des primates [1, 3, 6, 13, 16, 18, 21, 25, 27]. Cependant, peu de travaux s'intéressent au processus coracoïde, en dehors de celui de Martin et O'Brien [10]. Sur la partie distale du processus coracoïde (encore nommée lame du processus), s'insèrent trois muscles et cinq ligaments. Son orientation dans l'espace par rapport à d'autres structures scapulaires ou au squelette axial ne peut donc être aléatoire. Le travail présenté ici est une étude comparée de l'orientation et de la morphologie de la partie distale du processus coracoïde chez les hominoïdes, les atèles et les colobes, ainsi que chez trois hominidés fossiles. Les atèles et les colobes ont été intégrés dans cette étude, car ils présentent des convergences locomotrices avec certains hominoïdes non humains. Les processus coracoïdes des scapulas des hominidés fossiles sont-ils identiques à ceux des hommes modernes ? Les scapulas d'hominidés fossiles ayant conservé cette structure sont rares (au sein des hominidés, il existe uniquement trois scapulas néandertaliennes et une d'Homo ergaster, qui présentent encore un processus coracoïde), ce qui fait que nous ne pourrons pas conclure définitivement, mais proposerons des hypothèses qui seront confirmées ou infirmées lors d'études ultérieures.

\section{Matériel et méthodes}

Le matériel (tableau I) a été mesuré avec un dioptographe et un pied à coulisse et provient des collections du laboratoire d'anthropologie biologique du musée de l'Homme, du laboratoire d'anatomie comparée du Muséum national d'histoire naturelle, du musée royal d'Afrique centrale de Tervuren et du Mammals Group, Natural History Museum de Londres.

Tableau I. Effectif des scapulas étudié, C : captif, I : indéterminé, NC : non captif.

Table I. Numbers of studied scapulas, C: captive, I: inderterminate, NC: non-captive.

\begin{tabular}{lcrrr}
\hline Espèces (abréviation) & Spécimens & $\% \mathrm{C}$ & $\% \mathrm{I}$ & $\% \mathrm{NC}$ \\
\hline Homo sapiens & & & & \\
$\quad$ sapiens $(\mathrm{Hm})$ & 29 & & & \\
Pan troglodytes $(\mathrm{Pt})$ & 30 & 26,7 & 13,3 & 60,0 \\
Pan panicus $(\mathrm{Pp})$ & 19 & 0,0 & 0,0 & 100,0 \\
Gorilla & & & & \\
$\quad$ gorilla $(\mathrm{Gor})$ & 24 & 34,3 & 28,9 & 36,8 \\
Pongo pygmaeus $(\mathrm{Oo})$ & 18 & 16,6 & 29,2 & 54,2 \\
Hylobates sp. $(\mathrm{Gb})$ & 12 & 0,0 & 60,0 & 33,3 \\
Ateles sp. (At) & 25 & 8,0 & 64,0 & 28,0 \\
Colobus sp. $(\mathrm{Cl})$ & 2 (Tabun C1 & & & \\
Néandertalien & et Kebara droite) & & & \\
& 1 (KNM-WT & & & \\
Homo ergaster & $15000)$ & & & \\
&
\end{tabular}



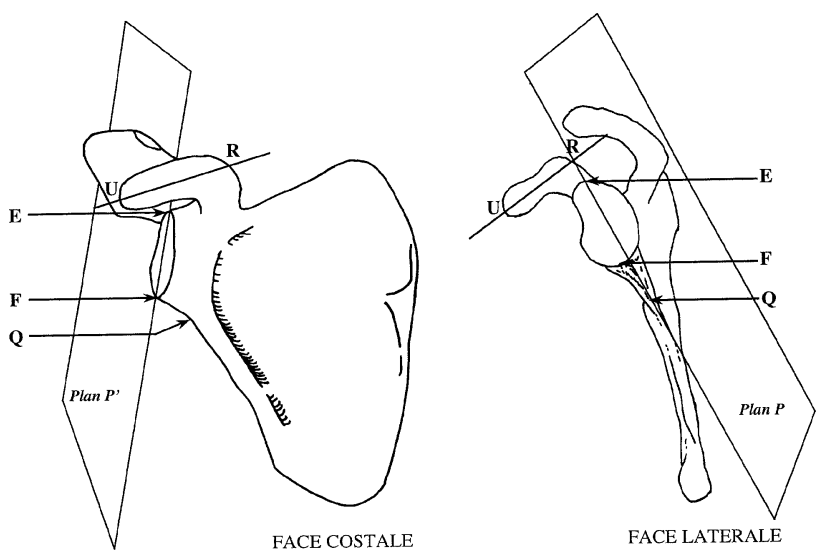

Figure 1. Points de référence.

Figure 1. Reference points.

Trois points définissent deux plans de référence $P$ et $P^{\prime}$, et deux autres le grand axe du processus coracoïde (figure 1). La projection de ces cinq points dans les deux plans de référence permet de connaître l'orientation du processus coracoïde par rapport à la cavité glénoïdale.

\subsection{Points et plans de référence}

- $E$ : point le plus saillant de la partie supérieure du sourcil glénoïdien [25].

- $F$ : point le plus éloigné du point $E$, sur le rebord glénoïdien [25].

- $Q$ : extension maximale du tubercule infraglénoïdal

en vue glénoïdale.

- $R$ et $U$ représentent le grand axe de la partie distale du processus coracoïde. $R$ est le point le plus saillant, à la jonction entre la lame du processus coracoïde et la base de cette dernière. $U$ est à l'extrémité distale de la lame du processus coracoïde. Il est sur l'axe passant par $R$ et par un point équidistant aux deux bords de la lame, au milieu de celle-ci.

- Plan $P$ : défini par les points $E, F$ et $Q$.

- Plan $P^{\prime}$ : perpendiculaire au plan $P$, passant par les points $E$ et $F$.

\subsection{Angles et indices}

\subsubsection{Orientation de la partie distale du processus coracoüde}

- Angle gléno-coracoïde- $p$ : projection de l'angle $(E F, R U)$ dans le plan $P$.

- Angle gléno-coracoïde- $p^{\prime}$ : projection de l'angle $(E F, R U)$ dans le plan $P^{\prime}$.

Les angles choisis ici ont pour but de préciser l'orientation de la partie distale du processus coracoïde par rapport à la scapula chez les primates. Cette structure étant complexe, d'autres angles ont été proposés [12,27], décrivant d'autres aspects du processus coracoïde.

\subsubsection{Longueur et robustesse de la partie distale du processus coracoïde}

- Indice coracoïdien : longueur $[R U] /$ longueur $[E F]$ $\times 100$.

- Indice de robustesse maximale : longueur $[R U] /$ largeur maximale du processus $\times 100$.

- Indice de robustesse minimale : longueur $[R U] /$ largeur minimale du processus $\times 100$.

Les largeurs minimale et maximale sont prises perpendiculairement à la longueur $[R U]$ et mesurées directement sur le processus coracoïde.

Ces trois indices décrivent succinctement la morphologie de la lame du processus coracoïde.

Les variables sont décrites par l'intermédiaire de statistique descriptive et complétées, le cas échéant, par des tests « $t »$, calculés avec le logiciel Statistica.

\section{Résultats}

La distribution des valeurs moyennes des angles gléno-coracoïde- $p$ et gléno-coracoïde- $p^{\prime}$ permet de distinguer deux groupes parmi les primates étudiés ici, l'homme (actuel et fossile), qui correspond aux valeurs angulaires les plus élevées (figures 2 et 3 , tableaux $I I$ et $V$ ), et les autres primates, où aucune espèce ne se différencie nettement. Autrement dit, l'orientation de la partie distale du processus coracoïde dans l'espace, par rapport à la cavité glénoïdale, n'est pas identique chez l'homme et l'ensemble constitué des autres hominoïdes, des atèles et des colobes. Chez les néandertaliens, l'orientation de l'extrémité distale du processus coracoïde par rapport à la scapula semble être identique à ce qui existe chez l'homme moderne. $\mathrm{Au}$ contraire, les valeurs des angles gléno-coracoïde$p$ et gléno-coracoïde- $p^{\prime}$ de KNM-WT 15000 sont en dehors de l'intervalle humain moderne (tableau $I V$ ). Cependant, cet individu était un adolescent [30] et la disparition des cartilages de conjugaison, non fossilisés, peut expliquer ces valeurs particulières, qui contrastent avec les autres caractères scapulaires qui sont tout à fait modernes [27, 30].

L'indice coracoïdien présente les valeurs les plus élevées chez les espèces brachiatrices, tels que les gibbons ou les atèles (figure 4, tableau III). En règle générale, plus l'animal est arboricole, plus l'indice coracoïdien augmente. Cette observation est confirmée par la très petite taille des processus coracoïdes des primates quadrupèdes terrestres tels que les babouins, alors qu'elle est nettement plus longue chez les primates quadrupèdes arboricoles, tels que les colobes [3, 27]. La valeur de l'indice coracoïdien de Tabun $\mathrm{C} 1$ est très proche de la moyenne de l'homme moderne, contrairement à celles de Kebara droite et de KNM-WT 15000 , qui correspondent à la limite inférieure de l'intervalle de l'homme moderne 
J.-L. Voisin / C. R. Acad. Sci. Paris, Sciences de la Terre et des planètes / Earth and Planetary Sciences 332 (2001) 299-306

Tableau II. Amplitudes de variation et valeurs moyennes (en degrés) des angles gléno-coracoïde- $p$ et gléno-coracoïde- $p^{\prime}$.

Table II. Ranges of variation and means (in degrees) of gleno-coracoid- $p$ and gleno-coracoid- $p^{\prime}$ angles.

\begin{tabular}{|c|c|c|c|c|c|c|}
\hline & \multicolumn{3}{|c|}{ Gléno-coracoïde- $p$} & \multicolumn{3}{|c|}{ Gléno-coracoïde- $p^{\prime}$} \\
\hline & $-2 \delta$ & moyenne & $+2 \delta$ & $-2 \delta$ & moyenne & $+2 \delta$ \\
\hline Homo sapiens sapiens & 66,5 & 81,9 & 97,4 & 45,9 & 72,5 & 99,0 \\
\hline Pan troglodytes & 34,5 & 48,9 & 63,3 & 11,3 & 31,6 & 52,0 \\
\hline Pan panicus & 39,5 & 58,0 & 77,7 & 28,3 & 45,1 & 61,8 \\
\hline Gorilla gorilla & 16,7 & 43,9 & 71,1 & 34,1 & 51,9 & 69,8 \\
\hline Pongo pygmaeus & 27,4 & 41,7 & 56,0 & 19,1 & 36,7 & 54,2 \\
\hline Hylobates sp. & 30,0 & 53,6 & 77,3 & 11,4 & 28,7 & 45,9 \\
\hline Ateles $s p$. & 25,8 & 44,2 & 62,6 & 13,8 & 32,4 & 51,1 \\
\hline Colobus sp. & 40,5 & 57,8 & 75,2 & 19,7 & 35,8 & 51,9 \\
\hline
\end{tabular}

Tableau III. Amplitudes de variation et valeurs moyennes des indices décrivant le processus coracoïde.

Table III. Ranges of variation and means of the index describing the coracoïd process.

\begin{tabular}{|c|c|c|c|c|c|c|c|c|c|}
\hline & \multicolumn{3}{|c|}{ Indice coracoïdien } & \multicolumn{3}{|c|}{ Indice de robustesse maximal } & \multicolumn{3}{|c|}{ Indice de robustesse minimal } \\
\hline & $-2 \delta$ & moyenne & $+2 \delta$ & $-2 \delta$ & moyenne & $+2 \delta$ & $-2 \delta$ & moyenne & $+2 \delta$ \\
\hline Homo sapiens sapiens & 110,8 & 124,9 & 139,0 & 28,1 & 34,9 & 41,8 & 24,5 & 31,5 & 38,5 \\
\hline Pan troglodytes & 121,4 & 145,0 & 168,6 & 28,3 & 36,4 & 44,5 & 24,2 & 29,6 & 35,0 \\
\hline Pan panicus & 110,4 & 135,1 & 159,9 & 36,3 & 45,1 & 54,0 & 30,3 & 37,7 & 45,1 \\
\hline Gorilla gorilla & 117,8 & 137,5 & 157,1 & 28,6 & 41,9 & 55,2 & 27,2 & 32,1 & 41,9 \\
\hline Pongo pygmaeus & 87,9 & 107,1 & 126,2 & 34,1 & 50,5 & 66,9 & 34,1 & 42,3 & 50,5 \\
\hline Hylobates sp. & 136,2 & 164,8 & 193,4 & 29,1 & 42,7 & 56,2 & 18,5 & 33,6 & 49,2 \\
\hline Ateles sp. & 140,7 & 179,7 & 219,3 & 20,8 & 25,7 & 30,6 & 19,1 & 22,5 & 25,7 \\
\hline Colobus sp. & 84,7 & 136,0 & 110,3 & 24,8 & 36,2 & 47,6 & 19,9 & 27,5 & 35,1 \\
\hline
\end{tabular}

Tableau IV. Caractéristiques du processus coracoïde de Kebara, Tabun C1 et KNM-WT 15000.

Table IV. Coracoid process characteristics of Kebara, Tabun C1 and KNM-WT 15000.

\begin{tabular}{lccccc}
\hline & $\begin{array}{c}\text { Angle } \\
\text { gléno-coracoïde- } p\end{array}$ & $\begin{array}{c}\text { Angle } \\
\text { gléno-coracoïde- } p^{\prime}\end{array}$ & $\begin{array}{c}\text { Indice } \\
\text { coracoïdien }\end{array}$ & $\begin{array}{c}\text { Robustesse } \\
\text { maximale }\end{array}$ & $\begin{array}{c}\text { Robustesse } \\
\text { minimale }\end{array}$ \\
\hline Kebara droite & 95,0 & 84,5 & 113,8 & 54,6 & 33,3 \\
Tabun C1 & 67,0 & 70,0 & 127,3 & 45,7 & 34,3 \\
KNM-WT 15000 & 59,5 & 38,0 & 110,7 & 45,2 & 25,8 \\
\hline
\end{tabular}

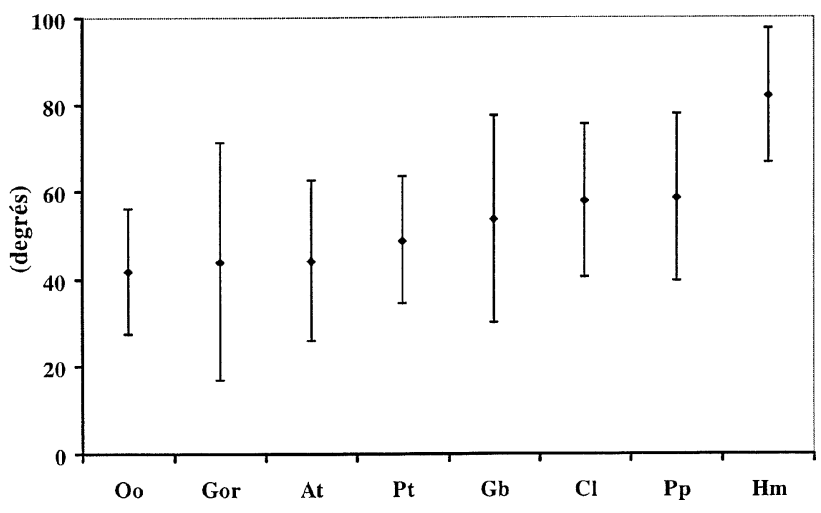

Figure 2. Moyenne et amplitudes de variation $( \pm 2 \delta)$ de l'angle gléno-coracoïde- $p$.

Figure 2. Mean and range of variation $( \pm 2 \delta)$ of the gleno-coracoid- $p$ angle.

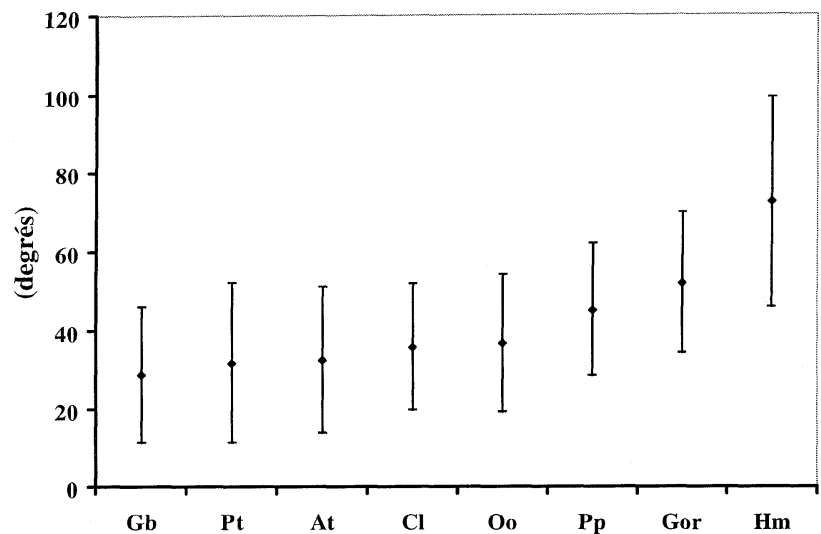

Figure 3. Moyenne et amplitudes de variation $( \pm 2 \delta)$ de l'angle gléno-coracoïde- $p^{\prime}$.

Figure 3. Mean and range of variation $( \pm 2 \delta)$ of the gleno-coracoid$p^{\prime}$ angle. 
Tableau V. Test- $t$ comparant les indices de robustesses minimales entre les colobes et les atèles.

Table V. $t$-test for the minimum robustness index between spider and colobus monkey.

\begin{tabular}{lccc}
\hline Groupe & $n$ & Moyenne & $\delta$ \\
\hline Colobe & 25 & 27,5 & 3,8 \\
Atèle & 12 & 22,5 & 1,7 \\
\hline
\end{tabular}

Valeur $t=4,8 ; \mathrm{ddl}=35 ;$ Prob $=0,00$.

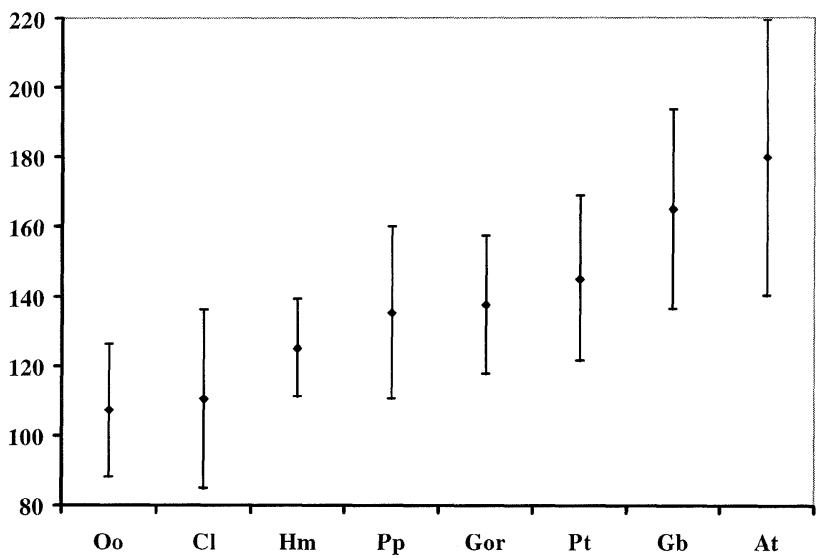

Figure 4. Moyenne et amplitudes de variation $( \pm 2 \delta)$ de l'indice coracoïdien.

Figure 4. Mean and range of variation $( \pm 2 \delta)$ of the coracoid index.

(tableau IV). Chez Kebara, cet indice est sous-estimé car, contrairement à Tabun $\mathrm{C} 1$, une partie importante du processus est manquante. La valeur de l'indice coracoïdien de KNM-WT 15000 n'est que légèrement sous-estimée car, bien que cette scapula ait perdu ses cartilages de conjugaison, ces derniers ne représentent pas une proportion importante de la longueur totale du processus.

La comparaison des valeurs moyennes des indices de robustesse du processus coracoïde permet de différencier deux groupes (figures 5 et 6 , tableau III) : d'une part, les atèles, qui présentent les processus coracoïdes les moins robustes, et, d'autre part, les hominoïdes et les colobes. Au sein de ce dernier groupe, il n'existe pas de sous-ensembles nettement distincts (figures 5 et 6, tableau III). Cependant, les colobes présentent une amplitude de variation importante ( $f$ gures 5 et 6 ) recouvrant en partie les valeurs des atèles (tableau V).

Les hominidés fossiles étudiés ici montrent des particularités par rapport à l'homme moderne. La robustesse coracoïdienne minimale de Tabun $\mathrm{C} 1$ et de Kebara droite présente des valeurs qui se situent à la limite inférieure de l'intervalle de variation de l'homme moderne et KNM-WT 15000 , une valeur plus faible que cette limite (tableau IV). Par ailleurs, la valeur obtenue pour Kebara droite est surestimée, car le processus coracoïde n'est pas complet. La



Figure 5. Moyenne et amplitudes de variation $( \pm 2 \delta)$ de l'indice de robustesse maximale.

Figure 5. Mean and range of variation $( \pm 2 \delta)$ of the maximum robustness index.

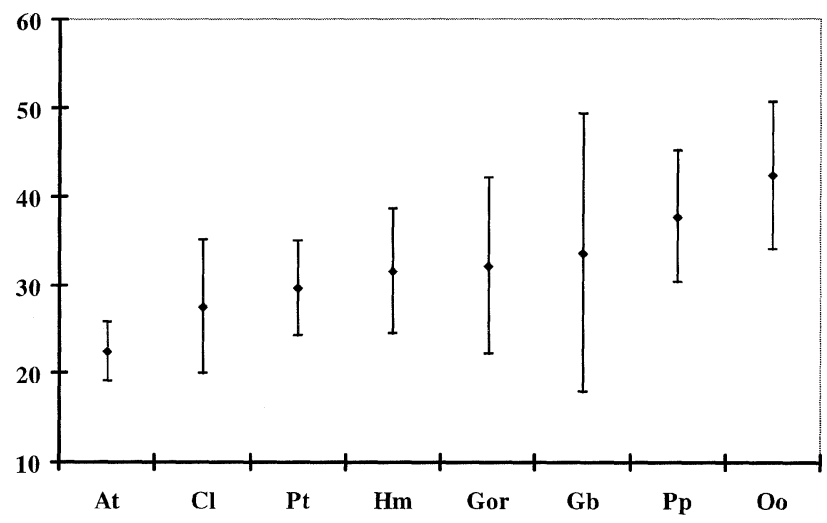

Figure 6. Moyenne et amplitudes de variation $( \pm 2 \delta)$ de l'indice de robustesse minimale.

Figure 6. Mean and range of variation $( \pm 2 \delta)$ of the minimum robustness index.

robustesse minimale est donc plus faible chez Kebara, Tabun C1 et KNM-WT 15000, que chez l'homme moderne.

La robustesse maximale tend à être plus importante chez KNM-WT 15000 , Tabun C1 et Kebara droite (tableau IV) que chez l'homme moderne. Chez Kebara, la valeur obtenue est surestimée, car le processus n'est pas complet, mais la robustesse devait malgré tout être importante.

\section{Discussion}

L'orientation dans l'espace de l'extrémité distale du processus coracoïde par rapport à la cavité glénoïdale permet de distinguer deux groupes, l'homme d'un côté et les autres hominoïdes, les atèles et les colobes de l'autre. Au contraire, il semble que son orientation par rapport au squelette axial soit constante $(f-$ gure 7). En effet, les scapulas des hominoïdes et des atèles sont situées dorsalement par rapport au thorax, 

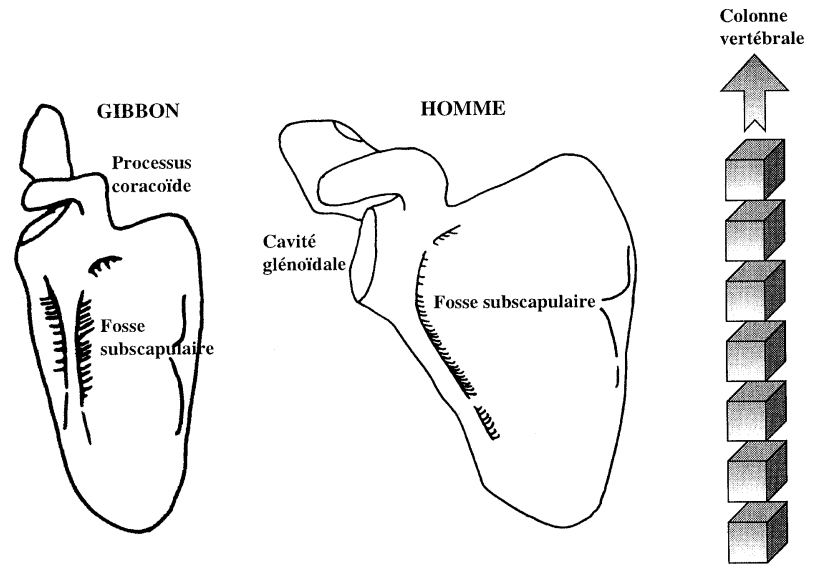

Figure 7. Position de la scapula par rapport au squelette axial. Noter l'orientation du processus coracoïde par rapport à la cavité glénoïdale et par rapport à la colonne vertébrale.

Figure 7. Position of the scapula in relation to the axial skeleton. Note the orientation of the coracoid process in relation to both glenoidal cavity and axial skeleton.

Tableau VI. Test- $t$ comparant les indices coracoïdiens entre atèles et gibbons.

Table VI. $t$-test for the coracoid index between spider monkeys and gibbons.

\begin{tabular}{lccc}
\hline Groupe & $n$ & Moyenne & $\delta$ \\
\hline Gibbon & 18 & 164,8 & 14,3 \\
Atèle & 12 & 179,7 & 19,8 \\
\hline
\end{tabular}

Valeur $t=-2,4 ; \mathrm{ddl}=28 ;$ Prob $=0,02$.

avec le bord médial parallèle à la colonne vertébrale lorsque l'individu est en position de repos, c'est-à-dire bras pendant chez l'homme [14-16]. Dans cette position, le processus coracoïde est dans un plan horizontal, perpendiculaire au squelette axial (figure 7). Ainsi, les différences constatées sur les angles glénocoracoïde- $p$ et gléno-coracoïde- $p^{\prime}$ entre l'homme et l'ensemble des hominoïdes non humains et des atèles seraient dues à l'orientation différente de la cavité glénoïdale entre ces deux groupes [16, 27]. En d'autres termes, l'orientation de l'extrémité distale du processus coracoïde par rapport à la cavité glénoïdale ne refléterait que l'orientation de cette dernière. Les colobes, au contraire, possèdent une scapula latérale au repos, associée à des déplacements quadrupèdes dominants, contrairement aux atèles [12]. Par ailleurs, lors des mouvements des bras, la scapula se déplace autour du thorax, notamment lors de la suspension de l'individu par les membres supérieurs. Dans ce cas de figure, la scapula des colobes prend sans doute une position similaire à celle qu'elle a chez les hominoïdes, d'autant plus que l'orientation de la cavité glénoïdale est proche entre les atèles et les colobes [27].

L'indice coracoïdien d'une espèce est d'autant plus grand qu'elle pratique le déplacement suspendu ( $f$ gure 4). En d'autres termes, plus les mouvements du bras en dehors du plan parasagittal sont fréquents, plus le processus coracoïde s'allonge. En effet, dans un cadre plus général, ce processus est très long chez les chiroptères [20], alors qu'il se limite à un bourrelet chez les mammifères strictement quadrupèdes, tels que les chevaux [9]. Partant de ce constat, la faible longueur du processus coracoïde chez les orangsoutans est étonnante. Elle est peut-être à mettre en relation avec la locomotion propre à cette espèce et au fonctionnement particulier de sa musculature scapulaire [23, 24], qui le distingue des autres hominoïdes, notamment des chimpanzés $[7,8]$.

Le processus coracoïde de l'atèle est, en moyenne, plus long que celui des gibbons (tableau VI, figure 4) car, comme chez les autres Atelinae et Alouattinae, il présente, à la jonction des parties distale et proximale, une excroissance qui se développe vers le haut et vers l'arrière [31]. Cette excroissance permet l'insertion d'un faisceau du muscle subclavier [31,32].

Chez les hominoïdes et les atèles, l'orientation similaire du processus coracoïde par rapport au squelette axial permet à celui-ci d'être toujours situé médialement et au-dessus de la tête humérale (figure 7). Ainsi, le processus coracoïde conserve sa fonction de bras de levier pour le coraco-brachial et la courte portion du biceps, quelle que soit la morphologie scapulaire. Le processus coracoïde permet alors de retrouver l'orientation anatomique d'un fragment de scapula chez un hominoïde fossile. L'augmentation de la longueur de cette structure renforce l'effet de bras de levier et permet d'avancer les insertions musculaires du coraco-brachial et de la courte portion du biceps (sauf chez le gibbon, où ce dernier s'insère sur l'extrémité proximale de l'humérus $[5,11])$, au-delà du bord antérieur de la clavicule. L'aspect quasi rectiligne des clavicules d'orangs-outans $[16,28]$ en vue crâniale expliquerait, en partie, le court processus coracoïde de leur scapula, en regard des autres hominoïdes. Une clavicule peu sinueuse nécessite un moindre allongement du processus pour qu'il soit projeté au-delà du bord antérieur de la clavicule. De même, les colobes possèdent un indice coracoïdien (figure 4) et une sinuosité claviculaire proche de ce qui existe chez les orangs-outans [27]. Par ailleurs, l'allongement du processus coracoïde ne peut être associé à la présence de la voûte acromio-coracoïdienne, puisque le ligament acromio-coracoïdien est absent chez Ateles et présent chez Pongo [4].

Le processus coracoïde présente un écart plus important entre la robustesse maximale et minimale chez les quelques néandertaliens et Homo ergaster qui le présentent encore que chez l'homme moderne. Cependant, Stewart [19] décrit le processus coracoïde de Shanidar 1 comme étant gracile et particulièrement mince, bien que ses données métriques montrent, au contraire, une robustesse importante. Trinkaus [22] 
J.-L. Voisin / C. R. Acad. Sci. Paris, Sciences de la Terre et des planètes / Earth and Planetary Sciences 332 (2001) 299-306

ne décrit pas cette structure, bien qu'il en donne deux mensurations. Mais elles ne correspondent pas à celles utilisées dans ce travail.

\section{Conclusion}

Ce travail montre que l'orientation dans l'espace du processus coracoïde par rapport à la cavité glénoïdale permet de distinguer deux groupes : l'homme moderne, d'un côté, et les autres hominoïdes, ainsi que les atèles et les colobes, de l'autre. Au contraire, le processus coracoïde semble orienté de manière similaire chez tous les hominoïdes et les atèles, lorsque le repère est pris par rapport au squelette axial. Cette disposition se retrouverait chez les colobes lors des phases de suspension. Ces deux derniers points nécessiteraient d'être confirmés par des études ultérieures, notamment par ciné-radiographie. Par ailleurs, la longueur de ce processus refléterait les capacités de mouvements des membres supérieurs en dehors du plan parasagittal et la morphologie claviculaire.
KNM-WT 15 000, Kebara et Tabun C1 présentent une orientation et une longueur du processus coracoïde, identiques à celles de l'homme moderne, ce qui traduit une disposition de la cavité glénoïdale par rapport au squelette axial, identique à celle de l'homme moderne ainsi que des contraintes sur le processus coracoïde similaire entre ces groupes humains.

$\mathrm{Au}$ contraire, il semblerait que le processus coracoïde de l'homme moderne présente une amplitude de variation moindre entre la robustesse minimale et maximale, que celle constatée chez les néandertaliens et Homo ergaster. Cette constatation, difficile à interpréter, pourrait être un critère de plus de différenciation de la scapula moderne par rapport à celle des autres groupes humains.

Quoi qu'il en soit, des études ultérieures sont nécessaires afin de confirmer ou d'infirmer les hypothèses énoncées dans ce travail. Il faudrait notamment découvrir plus de restes scapulaires fossiles et étudier le seul processus coracoïde connu d'australopithèque (Sts 7), afin d'apporter des résultats complémentaires aux travaux précédents $[2,17,26,29]$.

Remerciements. Je souhaite remercier ici le professeur H. de Lumley de m'avoir accueilli au sein de son laboratoire, ainsi que Mme M.-A. de Lumley, qui suit mes travaux avec intérêt. Je remercie aussi Mme B. Senut pour ses conseils avisés.

\section{Références}

[1] Ashton E.H., Oxnard C.E., Functional adaptations in the primate shoulder girdle, Proc. Zool. Soc. London 142 (1964) 49-66.

[2] Broom R., Robinson J.T., Schepers G.W.H., Sterkfontein ApeMan Plesianthropus, Trans. Mus. Mem. 4 (1950) 11-117.

[3] Ciochon R.L., Evolution of the Cercopithecoid forelimb. Phylogenetic and functional implication from morphometric analyses, Univ. Calif. Publ. Geol. Sci. 138 (1993) 1-251.

[4] Ciochon R.L., Corruccini R.S., The coraco-acromial ligament and projection index in Man and other anthropoïd Primates, J. Anat. 124 (1977) 627-632.

[5] Jouffroy F.-K., La musculature des membres chez les Lémuriens de Madagascar. Étude descriptive et comparative, Mammalia Suppl. 26 (2) (1962) 1-322.

[6] Larson S.G., New characters for the functional interpretation of primate scapulae and proximal humeri, Am. J. Phys. Anthtrop. 98 (1995) 13-35.

[7] Larson S.G., Stern J.T. Jr., EMG of scapulohumeral muscles in the chimpanzee during reaching and 'arboreal' locomotion, Am. J. Anat. 176 (1986) 171-190.

[8] Larson S.G., Stern J.T. Jr., Jungers W.L., EMG of serratus anterior and trapezius in the chimpanzee: scapular rotators revisited, Am. J. Phys. Anthtropol. 85 (1987) 71-84.

[9] Lessertisseur J., Saban R., Squelette appendiculaire, in : Grassé P.P. (Ed.), Traité de zoologie. Anatomie, systématique, biologie, t. XVI, fasc. 1, Masson, Paris, 1967, pp. 709-1078.

[10] Martin C.P., O'Brien H.D., The coracoïd process in the primate, J. Anat. Physiol. 73 (1939) 630-642.

[11] Miller R.A., Evolution of the pectoral girdle and forlimb in the primate, Am. J. Phys. Anthropol. 17 (1932) 2-56.
[12] Mittermeier R.A., Fleagle J.G., Locomotion and posture in Ateles and Colobus, Am. J. Phys. Anthropol. 45 (1976) 235-256.

[13] Ranke J., Zur Anthropologie des Schulterblattes, Korresp.-B1. Dtsch. Ges. Anthropol. Ethnol. Urgesc. 35 (1904) 139-144.

[14] Roberts D., Structure and function of the Primate scapula, in : Jenkins F.A. (Ed.), Primate Locomotion, Academic Press, New York, 1974.

[15] Sakka M., Morphologie évolutive de la tête et du cou chez l'Homme et les Grands Singes, in: Application aux Hominidés fossiles, t. I, Ensembles anatomiques et cervicaux, Cahiers de Paléoanthropologie, CNRS, Paris, 1985, 168 p.

[16] Schultz A.H., The skeleton of the trunk and limbs of higher primates, Hum. Biol. II (3) (1930) 303-438.

[17] Senut B., L'humérus et ses articulations chez les hominidés plio-pléistocènes, Cahiers de Paléoanthropologie, CNRS, Paris, 1981, $141 \mathrm{p}$.

[18] Sheat B.T., Scapula form and locomotion in chimpanzee evolution, Am. J. Phys. Anthropol. 70 (1986) 475-488.

[19] Stewart T.D., Neanderthal scapulae with special attention to the Shanidar Neanderthals from Iraq, Anthropos 57 (1962) 779-800.

[20] Strickler T.L., Functional osteology and myology of the shoulder in the Chiroptera, in : Contrib. Vert. Evol., Vol. 4, S. Karger, Basel, München, Paris, 1978, pp. 1-198.

[21] Taylor A.B., Scapula form and biomechanics in gorillas, J. Hum. Evol. 33 (1997) 529-553.

[22] Trinkaus E., The Shanidar neandertals, Academic Press, New York, 1983, $502 \mathrm{p}$.

[23] Tuttle R.H., Basmajian J.V., Electromyography of Pongid shoulder muscles and hominoid evolution. I. Retractor of the humerus and 'rotators' of the scapula, Yrbk. Phys. Anthropol. 20 (1977) 491497. 
J.-L. Voisin / C. R. Acad. Sci. Paris, Sciences de la Terre et des planètes / Earth and Planetary Sciences 332 (2001) 299-306

[24] Tuttle R.H., Basmajian J.V., Electromyography of pongid shoulder muscles. II. Deltoid, rhomboid and 'rotator cuff', Am. J. Phys. Anthropol. 49 (1978) 47-56.

[25] Vallois H.V., L'omoplate humaine. Étude anatomique et anthropologique, Bull. Mém. Soc. Anthropol. Paris, série $8^{\mathrm{e}}$ III (1932) 3-153.

[26] Vallois H.V., L'interprétation de l'omoplate de Plesianthropus transvaalensis, L'Anthropologie (Paris) 80 (2) (1976) 229-242.

[27] Voisin J.-L., L'épaule des Hominidés. Aspects architecturaux et fonctionnels, références particulières à la clavicule, thèse ( 2 volumes), Muséum national d'histoire naturelle, Paris, 2000, 442 p.

[28] Voisin J.-L., La clavicule humaine : adaptation à la station érigée ? Biom. Hum. Anthropol. 18 (2000) 15-22.
[29] Vrba E.S., A new study of the scapula of Australopithecus africanus from Sterkfontein, Am. J. Phys. Anthropol. 51 (1979) 117130.

[30] Walker A., Leakey R., in : Walker A., Leakey R. (Eds.), The Nariokotome Homo erectus skeleton, Springer Verlag, Berlin, 1993, $457 \mathrm{p}$.

[31] Youlatos D., Maîtrise de l'espace et accès aux ressources chez le singe hurleur roux (Alouatta seniculus) de la Guyane française - Étude morpho-fonctionnelle, thèse (2 volumes), Muséum national d'histoire naturelle, 1994, $141 \mathrm{p}$.

[32] Ziemer K., Functional morphology of forelimb joints in the woolly monkeys Lagothrix lagothricha, in: Contrib. Primatol., Vol. 14, S. Karger, Basel, 1978, 130 p. 